\title{
PAX9 polymorphism and susceptibility to sporadic non-syndromic severe anodontia: a case-control study in southwest China
}

Jing WANG ${ }^{1,2}$, Yuanzhi XU1 ${ }^{1}$, Jing CHEN $^{3}$, Feiyu WANG ${ }^{4}$, Renhuan HUANG ${ }^{4}$, Songtao WU ${ }^{4}$, Linjing SHU4, Jingyi QIU ${ }^{4}$, Zhi YANG ${ }^{5}$, Junjie XUE ${ }^{3}$, Raorao WANG ${ }^{1}$, Jilin ZHAO $^{6}$, Wenli LAl ${ }^{2}$

\author{
1- PhD, Department of Stomatology, Shanghai Tenth People's Hospital, Tongji University School of Medicine, Shanghai, P.R. China. \\ 2- PhD, MD, State Key Laboratory of Oral Diseases, Department of Orthodontics, West China Hospital of Stomatology, Sichuan University, Chengdu, Sichuan \\ Province, P.R. China. \\ 3- MD, State Key Laboratory of Oral Diseases, Department of Orthodontics, West China Hospital of Stomatology, Sichuan University, Chengdu, Sichuan \\ Province, P.R. China. \\ 4- B.S. Med, West China School of Stomatology, Sichuan University, Chengdu, Sichuan Province, P.R. China. \\ 5- PhD, Department of Oral \& Cranio-Maxillofacial Science, Shanghai Ninth People's Hospital, School of Medicine, Shanghai Jiaotong University, P.R. China. \\ 6- PhD, Department of Orthodontics, Hospital of Stomatology, Medical School, Nanjing University, Nanjing, Jiangsu Province, P.R. China.
}

Corresponding address: Wenli Lai - State Key Laboratory of Oral Diseases, Department of Orthodontics - West China Hospital of Stomatology, Sichuan University - NO. 14 - Section 3 - Ren Min Nan Road - Chengdu - 610041 - P.R. China - Phone: 86-28-85501442 - Fax: 86-28-85582167 - e-mail: wenlilai@ hotmail.com

Received: January 29, 2013 - Modification: March 25, 2013 - Accepted: April 24, 2013

\section{ABSTRACT}

$\mathrm{O}$ ur research aimed to look into the clinical traits and genetic mutations in sporadic non-syndromic anodontia and to gain insight into the role of mutations of $P A X 9, M S X 1$, AXIN2 and EDA in anodontia phenotypes, especially for the PAX9. Material and Methods: The female proband and her family members from the ethnic Han families underwent complete oral examinations and received a retrospective review. Venous blood samples were obtained to screen variants in the PAX9, MSX1, AXIN2, and EDA genes. A case-control study was performed on 50 subjects with sporadic tooth agenesis (cases) and 100 healthy controls, which genotyped a PAX9 gene polymorphism (rs4904210). Results: Intra-oral and panoramic radiographs revealed that the female proband had anodontia denoted by the complete absence of teeth in both the primary and secondary dentitions, while all her family members maintained normal dentitions. Detected in the female proband were variants of the PAX9 and AXIN2 including A240P (rs4904210) of the PAX9, c.148C >T (rs2240308), c. 1365A > G (rs9915936) and c.1386C > T (rs1133683) of the AXIN2. The same variants were present in her unaffected younger brother. The $P A X 9$ variations were in a different state in her parents. Mutations in the MSX1 and EDA genes were not identified. No significant diferences were found in the allele and genotype frequencies of the PAX9 polymorphism between the controls and the subjects with sporadic tooth agenesis. Conclusions: These results suggest that the association of $A 240 P$ with sporadic tooth agenesis still remains obscure, especially for different populations. The genotype/phenotype correlation in congenital anodontia should be verified.

Key words: PAX9 transcription factor. Polymorphism. Non-syndromic Anodontia. Phenotype. Genotype.

\section{INTRODUCTION}

Tooth agenesis is a common development anomaly in humans, which includes two types, syndromic or non-syndromic. The non-syndromic tooth agenesis with significant phenotypic variability has been classified as either sporadic or familial, which can be inherited in an autosomal-dominant, autosomal-recessive, or X-linked mode ${ }^{7,32}$. Anodontia (OMIM 206780) is the term conventionally used in cases where teeth are completely absent ${ }^{19}$. Sporadic non-syndromic anodontia not involving associated abnormalities is extremely rare. Some case reports have suggested that anodontia of permanent teeth 
is an expression of the homozygote state of the gene resulting in the pegged or missing maxillary incisor $^{9,31}$. Genetic and environmental factors may be of etiologic importance to this anomaly ${ }^{3,15}$. Brook ${ }^{1}$ (2009) considered that complex interactions between genetic, epigenetic and environmental factors caused dental anomalies during the long process of dental development, which is multi-factorial, multilevel, multidimensional and progressive over time. In other words, the phenotype often reveals variations between affected individuals in the same family as a result of the multi-directional role of genetic, epigenetic and environmental factors in space and time. A previous study ${ }^{30}$ showed that mutations or polymorphisms of the PAX9 (OMIM 167416), MSX1 (OMIM 142983), AXIN2 (OMIM 604425), and EDA (OMIM 300451) ${ }^{25}$ genes were responsible for isolated/non-syndromic oligodontia. The reasons are still unknown for the majority of the congenital sporadic forms of missing teeth in humans. Gene-gene interactions may underly a significant number of these cases. Numbers of studies have shown that mutations in the PAX9 or MSX1 cause non-syndromic oligodontia. Both the $P A X 9$ and MSX1 are transcription factors expressed in the dental mesenchyme during the initiation of tooth development. As a member of the paired box domain gene family, $P A X 9$ regulates the cellular pluripotency and differentiation during embryonic patterning and organogenesis ${ }^{13,28}$. Its defects are associated with agenesis of the permanent molars, mostly including the maxillary first molars, maxillary second molars, and mandibular second molars ${ }^{32}$. MSX1 belongs to the muscle segment homeobox family and is essential for the switch in odontogenic potential from the epithelium to the mesenchyme ${ }^{17,18}$. Mutations in the MSX1 have been identified to be responsible for the missing second premolars and third molars. AXIN2 (17q23-q24), which is known as a negative feedback regulator of the Wnt-signaling pathway, is expressed by the ectomesenchyme and regulates early organ differentiation and development. Mutations in the AXIN2 lead to tooth agenesis affecting mostly the permanent teeth, containing the permanent molars, the lower incisors, and the upper lateral incisors in humans. The EDA gene (MIM \#300451), encoding the protein ectodysplasin-A $(E D A)$, is a type II transmembrane protein with a C-terminal TNF homology domain consisting of 10 predicted anti-parallel $\beta$-sheets linked by variable loops. It is identified associated with syndromic tooth agenesis that underlies $\mathrm{X}$-linked hypodontia ectodermal dysplasia (XLHED; MIM \#305100) and the mutation analysis of the EDA gene in people with isolated oligodontia were recommended in some studies $4,8,26,33$.

Until now, we do not have enough knowledge about the importance of genes in the etiology of oligodontia in the Chinese population, although there have been some mutations identified, such as c. $662 \mathrm{C}>\mathrm{A}$ and c. $347 \mathrm{C}>\mathrm{G}$ of MSX1 for non-syndromic autosomal-dominant oligodontia ${ }^{32}$, heterozygous C139T transition in PAX934, and so on. Recently, two polymorphisms (rs2073244, rs2073246), which were located in the promoter region of the $P A X 9$ gene, were suggested to influence the risk of sporadic tooth agenesis in a Caucasian population ${ }^{24}$. However, it was reported that p.Ala240Pro in the $P A X 9$ might not be associated with tooth agenesis in a case-control study ${ }^{22}$. Therefore, whether the variants of PAX9 have an effect on the tooth agenesis in different populations is still puzzling.

In the current study, we investigated the mutations in the PAX9, MSX1, AXIN2 and EDA genes in a Chinese family and evaluated the association of gene variation and epigenetic factors with sporadic non-syndromic anodontia. Additionally, we performed genotyping analyses of a SNP (rs4904210) of the PAX9 in subjects with sporadic tooth agenesis (cases) and 100 healthy controls in a Chinese population to test the hypothesis whether the variant A240P (rs4904210) of the PAX9 gene is associated with susceptibility to tooth agenesis in the Chinese population.

\section{MATERIAL AND METHODS}

All these subjects were fully informed of the study and consent was obtained from each of them. The research was approved by the Ethics Committee of West China Hospital of Stomatology, Sichuan University (2009022). All participants in the study were ethnic Han Chinese from Chengdu City or the surrounding regions in southwest China.

\section{Study individuals}

The female proband from ethnic Han families was referred to the Department of Orthodontics, West China Hospital of Stomatology, Sichuan University (Chengdu, China). The pedigree of the family was constructed by extended interviews. Retrospective data were reviewed and panoramic radiographs were obtained to confirm the diagnosis of tooth agenesis for the proband. Panoramic radiographs were also performed for other family members to assess the dental development. These 4 subjects (including the proband) underwent complete oral examinations with a thorough clinical investigation of other ectodermal originated tissues, including the skin, hair, nails, sweat glands, ears and eyes.

\section{Case-control study}

A total of 50 subjects with sporadic tooth agenesis (excluding the third molar) and 100 healthy controls were consecutively recruited from the West China Hospital of Stomatology, Sichuan 
University (Chengdu, China) from October 2009 to November 2011. Retrospective data were reviewed and panoramic radiographs were obtained to confirm the diagnosis of tooth agenesis for the patients. Every participant underwent complete oral examinations with a thorough clinical investigation of other ectodermal originated tissues, including the skin, hair, nails, sweat glands, ears and eyes by an experienced doctor. The characteristics of the subjects were listed in Figure 1. The exclusion criteria were as follows: 1 . with positive family histories; 2. with non-dental abnormalities; 3. with tooth agenesis resulted from acquired reasons, such as extraction, trauma, or others.

\section{Mutation screening and DNA sequencing}

Peripheral blood samples were obtained. DNA was extracted from blood spots by the Chelex-100 (Bio-Rad Laboratories, Hercules, California, USA)

\begin{tabular}{|l|c|}
\hline \multicolumn{1}{|c|}{ Group } & Number \\
\hline Total of patients & 150 \\
\hline Non-syndromic agenesis patients & male 13 \\
female 31
\end{tabular}

Figure 1- Number of individuals with dental agenesis on filter papers (BFC 180, Whatman International, Maidstone, UK). The exons 1-4 of the PAX9, exons 1-2 of the MSX1, exons 2-11 of the AXIN2 and exons 1-8 of the EDA were sequenced except for the intronic and promoter sequences of these genes. Primers for the PAX9, MSX1, AXIN2 and EDA genes designed through the Oligo Primer Analysis Software were made by Dingan Biotech (Shanghai, China) ${ }^{10}$ (Figure 2 ). The $50 \mu L \mathrm{PCR}$ reaction was carried out with the use of PCR beads, $0.3 \mu \mathrm{M}$ (each) primer, $5 \mathrm{ng} / \mathrm{ul}$ template DNA, $2.5 \mathrm{U}$ Taq DNA Polymerase (Takara Biotechnology, Dallian, Liaolin, China), $0.3 \mu \mathrm{M}$ of dNTP final concentration, $2 \mathrm{mmol} / \mathrm{L}$ of $\mathrm{MgCl}_{2}$ and sterile distilled water. The amplification procedure contained $5 \mathrm{~min} 94^{\circ} \mathrm{C}$ denaturation, followed by 35 cycles of $30 \mathrm{sec} 94^{\circ} \mathrm{C}, 30 \mathrm{sec} 56^{\circ} \mathrm{C}$, and $1 \mathrm{~min}$ $72^{\circ} \mathrm{C}$, and $7 \mathrm{~min} 72^{\circ} \mathrm{C}$ extension using the $\mathrm{ABI} 9700$ thermal cycles (Applied Biosystems, Foster City, California, USA). The PCR products were subjected to electrophoresis in a $2 \%$ agarose gel and purified by the BigDye Terminator Kit for analysis. Purified double-stranded PCR products were analyzed on the 3730 DNA Analyzer (Applied Biosystems, Foster City, California, USA). The sequencing results of the PAX9, MSX1, AXIN2 and EDA genes were compared with the previously published human genome in the NCBI GeneBank by Using the Auto Assemble software. (Gene bank accession: PAX9, NC_000014.837126773-37147012; MSX1, NG_008121.1；AXIN2, NG_012142.1; EDA, NC_000086.697170945-97596099.)

\section{Statistical analysis}

To estimate the associations between the

\begin{tabular}{|c|c|c|c|c|}
\hline Primer name $\left.\mathbf{( 5}^{\prime} \rightarrow \mathbf{3}^{\prime}\right)$ & Primer sequence & Size $\mathbf{( b p )}$ & Annealing temperature ${ }^{{ }^{\circ} \mathbf{C}}$ & PCR \\
\hline Exon1 F1 & CCACTGTCGCGCAGGAAC & 631 & 67.3 & Nested PCR \\
\hline Exon1 R1 & TTGGCTCGAGGTTGCTGA & 631 & 64.8 & Nested PCR \\
\hline Exon2 F1 & GGGCTCAGGCTTTAGACAC & 502 & 61.7 & Nested PCR \\
\hline Exon2 R1 & TTTCAAGCGATTCTCCTGC & 502 & 63.3 & Nested PCR \\
\hline Exon3 F1 & GACCCTTGGCTGTGAGACTC & 237 & 63.6 & Nested PCR \\
\hline Exon3 R1 & ACAAAAATCGCACTCTTGAT & 237 & 59.5 & Nested PCR \\
\hline Exon4 F1 & AATCCCAGTTACTCCAGAGGC & 477 & 64.8 & Nested PCR \\
\hline Exon4 R1 & ACTGGGCTAGGAGATCTGCAT & 477 & 65.2 & Nested PCR \\
\hline Exon5 F1 & GGCCCACTGAAGATGAAG & 446 & 59.8 & Nested PCR \\
\hline Exon5 R1 & GGCAAGACACCCTTTCCT & 446 & 61.4 & Nested PCR \\
\hline Exon6 F1 & CAGTAACATCCCAAGACAGG & 347 & 59.6 & Nested PCR \\
\hline Exon6 R1 & CAGTAGAGGGCATGATGGAG & 347 & 62.3 & Nested PCR \\
\hline Exon7 F1 & TGGCAGCTGCTTTACAAAC & 435 & 61.8 & Nested PCR \\
\hline Exon7 R1 & ACCCAAAGCAGGAAGTTAG & 435 & 59.1 & Nested PCR \\
\hline Exon8 F1 & GCCAGCTAGCACGCCTTC & 566 & 66.2 & Nested PCR \\
\hline Exon8 R1 & GGCCTTGTCACCCTGGAG & 674 & 65 & Nested PCR \\
\hline
\end{tabular}

Figure 2- The sequence of EDA Primers 
PAX9 polymorphism (A240P) and the risk of tooth agenesis, the difference in the allele and genotype of the PAX9 polymorphism was evaluated by the chi-square and Fisher exact tests to compare the test subjects and controls. All statistical tests were analyzed using the SPSS software (SPSS 17.0, IBM, New York, NY, USA). Differences in the frequencies of each polymorphism between the test subjects and controls were considered statistically significant if $\mathrm{P} \leq 0.05$.

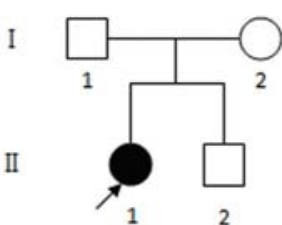

A

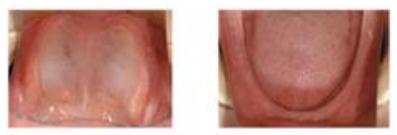

B

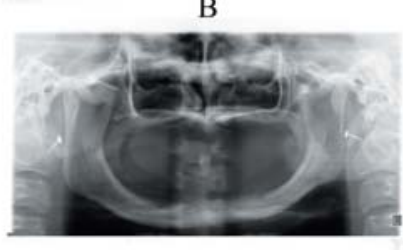

C
Figure 3- A: Pedigree of the female proband's family. B: Tooth phenotype of the female proband with severe nonsyndromic anodontia. C: Panoramic radiograph showed that the female proband (II - 1) at 21 years lacked all teeth

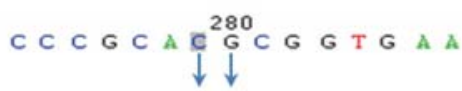

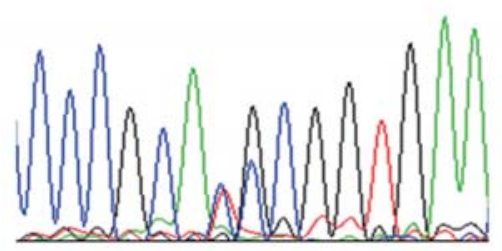

Proband II-1

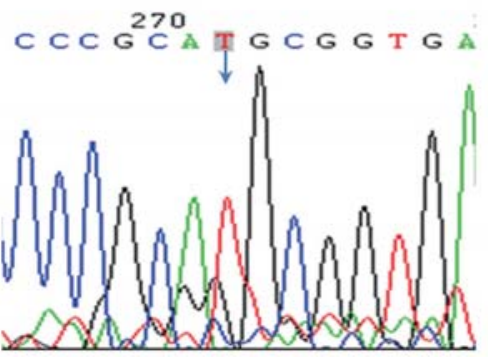

$\mathrm{I}-2$

\section{RESULTS}

\section{Clinical diagnosis/phenotype}

There was no history of tooth agenesis/anodontia in this family. Intra-oral and panoramic radiographs revealed that the female proband had anodontia denoted by the complete absence of teeth in both the primary and secondary dentitions (Figure 3 ), while all her family members maintained normal dentitions. No abnormalities were revealed in

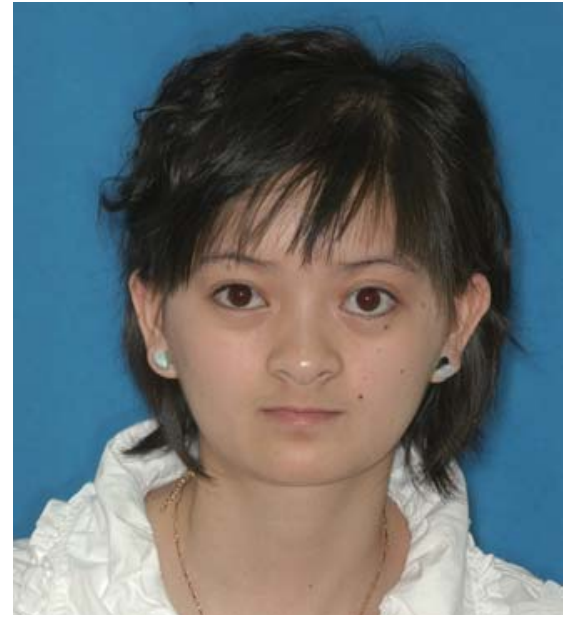

Figure 4- Maxillofacial appearance of the proband with severe dental agenesis. There is no other maxillofacial appearance defect or other unnormal
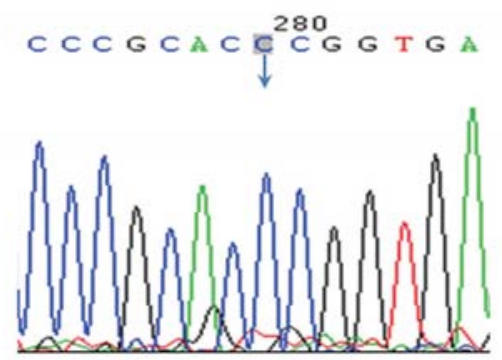

I-1
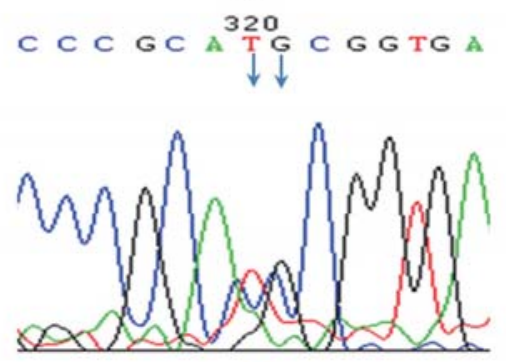

II-2

Figure 5- DNA-sequencing chromatograms of exon3 of PAX9 in the female proband (II-1) and her family members A variant of PAX9 gene $\{\mathrm{G} 718 \mathrm{C}$ ( $\mathrm{rs} 4904210)$ \} was detected in the heterozygous state in the female proband (II-1). The same variant in the homozygous state was present in her father (I-1) and heterozygous state in her young brother (II-2), but not in her unaffected mother (I-1) 
the sweat glands, hair, and nails through clinical examination of the proband (Figure 4) and her family members. Additionally, the distance between the orbits or eyebrows, and the development of the

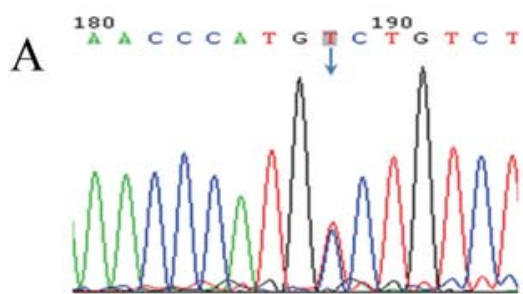

Proband II-1
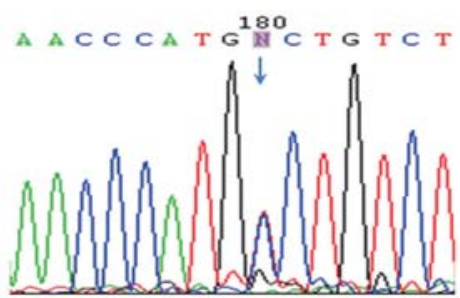

$\mathrm{I}-2$

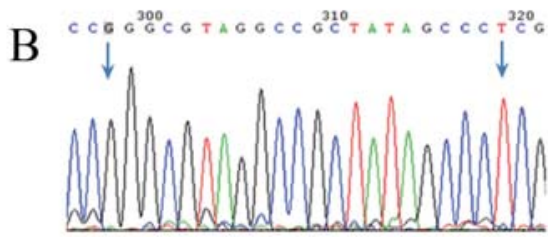

Proband II-1

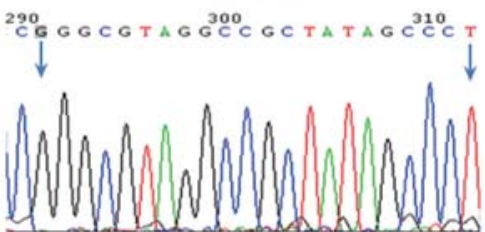

I-2 eyebrow bone were genetically normal. No other clinical features indicating a syndrome or systemic disorder were found.

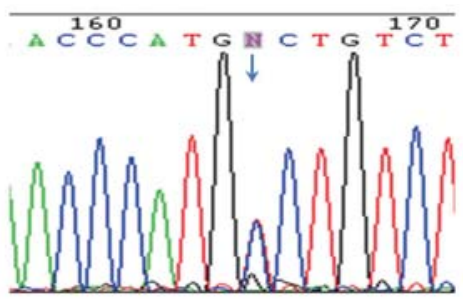

$\mathrm{I}-1$

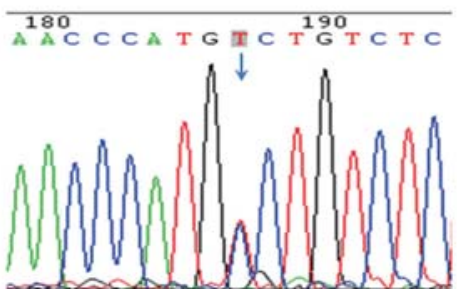

II-2

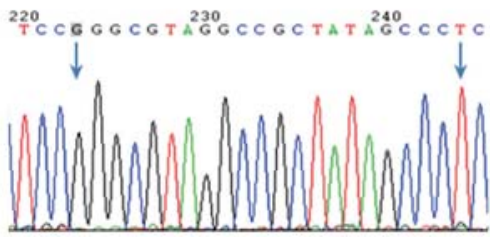

I-1

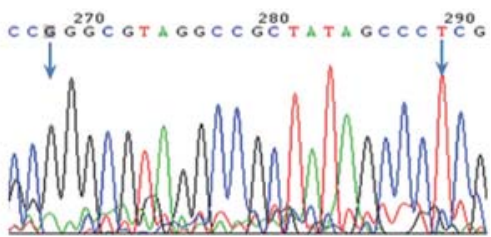

II-2

Figure 6- DNA-sequencing chromatograms of exon2 of AXIN2 in the female proband (II-1) and her family members A. c. $148 C>T$ (rs2240308) in AXIN2 gene was detected in the heterozygous state in the female proband (II-1), and the same variant was present in her unaffected younger brother (II-2) and her parents.

B. c. 1365A>G (rs9915936) and c.1386C>T (rs1133683) of AXIN2 gene were found in the homozygous state in the female proband (II-1), and the same variant was present in her unaffected family members in the homozygous state

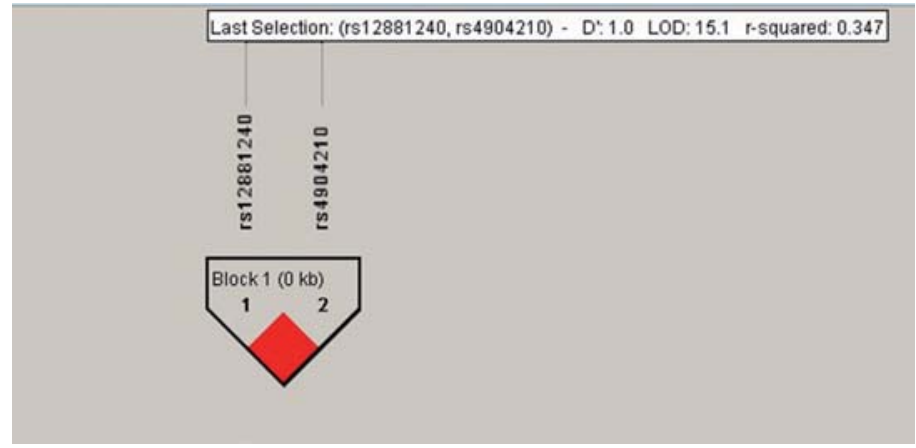

Figure 7- Pairwise linkage disequilibrium (LD) between the two PAX9 single nucleotide polymorphisms (SNPs), determined using HAPLOVIEW software. The strength of LD between the two SNPs was measured with the D' statistic. The numbers in the boxes are the D' value 


\section{Mutation analysis}

Variants of the PAX9 and AXIN2 were detected in the female proband, including G718C (p.Ala240Pro) in the exon3 of the PAX9, c.148C>T (p.Pro50Ser) in the exon2, c.1365A $>$ G (p.Pro455Pro) in the exon6 and c.1386C>T (p.Pro462Pro) in the exon6 of the AXIN2. The same variants were present in her unaffected younger brother (Figures 5,6). The
$P A X 9$ variations were in a different state in her parents (Figures 7). Mutations in the MSX1 and EDA genes were not identified in either the proband or her family members.

Results of association with the PAX9 polymorphism with tooth agenesis

No significant association with tooth agenesis

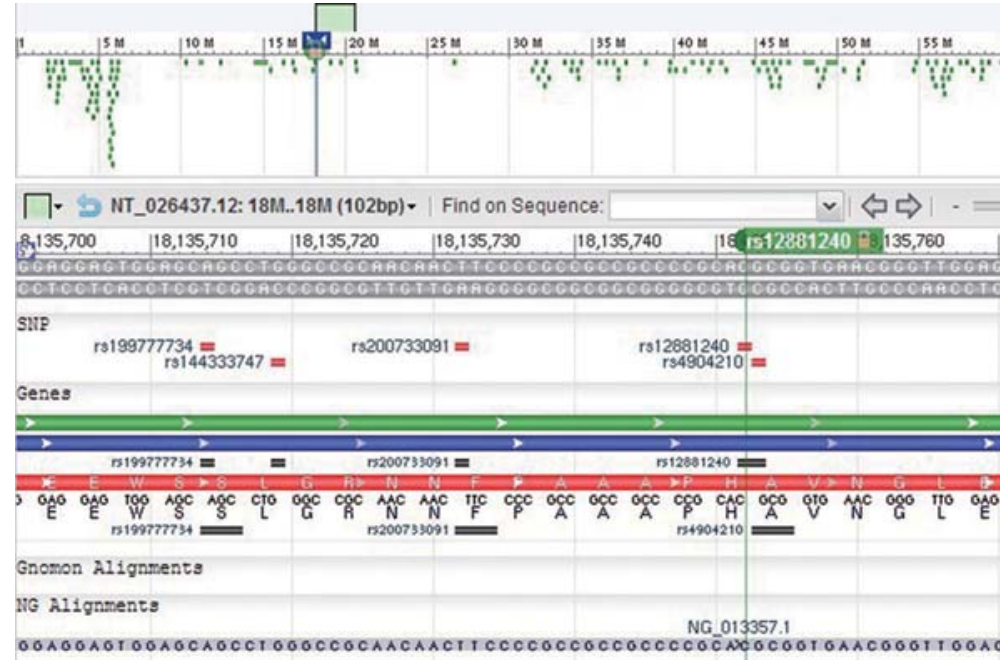

Figure 8- The gene chromatograms of c.717C > T (rs12881240) single nucleotide polymorphisms (SNPs) in PAX9

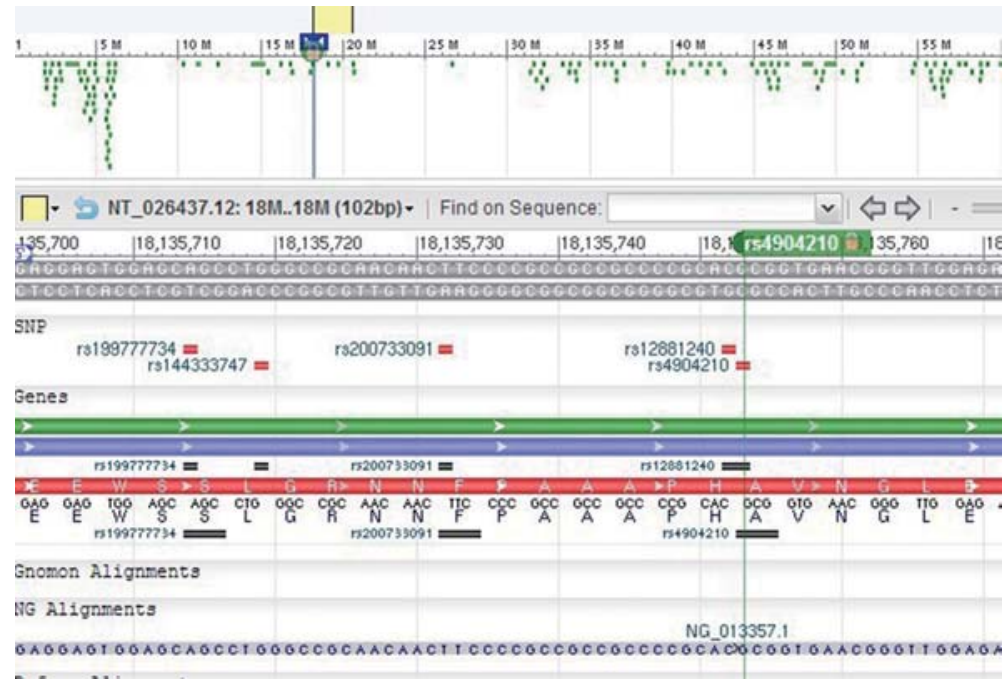

Figure 9- The gene chromatograms of c.718G >C (rs4904210) single nucleotide polymorphisms (SNPs) in PAX9

Table 1- Difference analysis of genotype (The differences of two constituent ratio analysis, conducting chi-square statistics) Sample size: 150

\begin{tabular}{cccccccc}
\hline & & \multicolumn{2}{c}{ Patient } & \multicolumn{2}{c}{ Control } & $\boldsymbol{X}^{2}$ value & $\boldsymbol{P}$ value \\
genotype & & N & proportion(\%) & N & proportion(\%) & \\
\hline C717T (rs12881240) & C & 70 & 70 & 148 & 74 & 0.537 & 0.464 \\
& T & 30 & 30 & 52 & 26 & & \\
G718C (rs4904210) & G & 52 & 52 & 104 & 52 & 0 & 1 \\
\hline & C & 48 & 48 & 96 & 48 & \\
\hline
\end{tabular}


Table 2- Difference analysis of gene frequency (the differences of two constituent ratio analysis, conducting chi-square statistics) Sample size: 150

\begin{tabular}{|c|c|c|c|c|c|c|}
\hline \multirow[b]{2}{*}{ genotype } & \multicolumn{2}{|c|}{ Patient } & \multicolumn{2}{|c|}{ Control } & \multirow[t]{2}{*}{$x^{2}$ value } & \multirow[t]{2}{*}{$P$ value } \\
\hline & $\mathbf{N}$ & Proportion (\%) & $\mathbf{N}$ & Proportion (\%) & & \\
\hline C717T (rs12881240) & & & & & 3.194 & $0.200^{*}$ \\
\hline $\mathrm{C} / \mathrm{C}$ & 25 & 50 & 51 & 51 & & \\
\hline $\mathrm{C} / \mathrm{T}$ & 20 & 40 & 46 & 46 & & \\
\hline $\mathrm{T} / \mathrm{T}$ & 5 & 10 & 3 & 3 & & \\
\hline G718C(rs4904210) & & & & & 0.503 & 0.778 \\
\hline $\mathrm{C} / \mathrm{C}$ & 10 & 20 & 17 & 17 & & \\
\hline $\mathrm{G} / \mathrm{C}$ & 28 & 56 & 62 & 62 & & \\
\hline $\mathrm{G} / \mathrm{G}$ & 12 & 24 & 21 & 21 & & \\
\hline
\end{tabular}

for alleles of the polymorphism (A240P) (Figures $8,9)$ was observed between the test subjects and controls $(P=1)$ (Table 1 and Figure 7 ). In addition, the genotype frequencies seemed to distribute no differences $(P=0.778)$ (Table 2$)$.

\section{DISCUSSION}

The female proband had anodontia denoted by the complete absence of teeth in both the primary and secondary dentitions, while all her family members maintained normal dentitions. A known mutation (G718C transversion) in the exon 3 of the $P A X 9$ resulted in the corresponding mutant $A 240 P$ in the female proband and her family members ( 1 - 1 , II - 2), whereas this mutation was not detected in her mother ( $\mathrm{I}-2)$. In the current research, there were no mutations in the MSX1 and EDA genes in the proband and her family members. Mostowska, et al. ${ }^{14}$ (2006) suggested that the variants c. $148 \mathrm{C}>\mathrm{T}, \mathrm{c} .1365 \mathrm{~A}>\mathrm{G}$ and c. $1386 \mathrm{C}>\mathrm{T}$ of $A X I N 2$ had no significant effect on the risk of tooth agenesis in a case-control study performed on 55 subjects with tooth agenesis and 102 healthy controls. There was yet no family history of these indications in the families. Human PAX9, highly conserved, consists of coding and non-coding regions. A240P is located in the coding region and probably contributes to structural and functional changes of the protein. This mutation in sporadic oligodontia was reported as polymorphic in many African, American, and European subpopulations. PaixãoCôrtes, et al. ${ }^{21}$ (2011) detected a complex involving the $P A X 9$ related to some dental morphological differences between Sub-Saharan Africans and non-Africans, which is probably associated with the different patterns of evolution in Africa and in other continents. Pawlowska ${ }^{23}$ (2010) found an A240P mutation in sporadic oligodontia with all the molars lacking. Another study ${ }^{10}$ reported a Chinese proband missing most of the anterior teeth had an A240P in the PAX9. However, it did not establish the presence or absence of an association of this mutation with the PAX9 associated phenotype. In a case-control study performed on 102 subjects with tooth agenesis and 116 healthy controls ${ }^{22}$, Pan, et al. ${ }^{22}$ (2008) suggested that the A240P mutation with $P A X 9$ had no significant effect on the risk of tooth agenesis. In the present study, we investigated the associations between the A240P PAX9 gene and sporadic tooth agenesis in a southwestern Han Chinese population, which yet found no evidence for A240P on the risk of sporadic tooth agenesis in our population. It seemed that A240P might have no susceptibility with sporadic tooth agenesis for Chinese people. Otherwise, a pedigree study of tooth agenesis indicated that the mutations in the $P A X 9$ gene showed the third molars missing ${ }^{12}$. The rejection of the sample missing the third molar is also the reason why we got the negative results. What we can see from this is that the association of A240P with sporadic tooth agenesis still remains obscure, especially for different populations.

Among the various factors of tooth agenesis (genetic factors, environmental factors, radiotherapy, and so on $)^{28}$, the genetic factor is known as an important role in the development of tooth agenesis. It is shown that dental agenesis may be caused by several independent defective genes, acting alone or in collaboration with others. Kangas, et al. ${ }^{11}$ (2004) reported that dental anomalies (the size and shape of teeth, tooth agenesis or supernumerary teeth) were determined by a set of genes in tooth development. PAX9 and AXIN2 mutations have been ruled out in a number of instances with severe oligodontia reported by Gerits, et al. ${ }^{6}$ (2006). Their mutations, however, partly account for the development of tooth agenesis ${ }^{2,5,29}$. However, our research suggested other genes were not yet associated with tooth agenesis. Some critical non-coding regions (eg. intronic and promoter sequences) of the PAX9, MSX1, AXIN2 and $E D A$ or changes in protein-protein interactions between these genes implicated it may attribute 
to anodontia in this family. Another possibility is a change in protein-protein interactions between the PAX9 and MSX1. Developmental studies have shown that the $P A X 9$ and $M S X 1$ are co-expressed in the mesenchyme during the bud stage of tooth development. Gerits, et al. ${ }^{6}$ (2006) found in vivo that mutated $M S X 1$ and $P A X 9$ proteins had alterations in their protein spatial structure, thereby influencing their thermo-stability and/or the threedimensional folding, which disturbed the normal functional activities of the mutant protein and changed its DNA-binding capacity and interactions with other transcriptional factors. Their expression seems to be requisite for the expression of BMP4 that plays an important role in tooth development ${ }^{25}$. Paixão-Côrtes, et al. ${ }^{20}$ (2011) suggested that the derived A240P PAX9 is related with third molar agenesis and that it may have a recessive pattern of inheritance with variable expressivity. On the other hand, one MSX1 gene derived allele appeared in agenesis affected individuals only. In conclusion, common variants located out of the DNA binding domain of these two transcription factor genes can also be related to tooth agenesis. Another study found direct evidence for a genetic interaction between heterozygous PAX9 and MSX1 loss-offunction mutations which result in oligodontia in mice $^{16}$. Thus, we suppose that interaction between the PAX9 mutation and the MSX1 may be another reason for the anodontia of the probed.

The female probed lacked all the teeth, while her younger brother with the same gene variants of the PAX9 and AXIN2 did not present any developmental abnormalities in the dentition (Figures 4,5). The etiology in these cases most probably consists of multiple factors: not only mutations found in different genes but also other factors such as epigenetic regulation and environmental factors that may result in different phenotypes. In our study, it may be considered that the epigenetic regulation is a factor that results in the different phenotypes between the female probed and her brother with the same gene mutations. The definition of epigenetic can refer to the addition or removal of methyl groups to DNA or acetylation of histones, while the broader definition is an alteration in gene expression without changes in nucleotide sequencing, including interaction between cells at a tissue level ${ }^{1,27}$. Previous studies support the view that epigenetic factors can influence the number or position of affected teeth, although there is a relatively strong genetic basis to missing or extra teeth ${ }^{27}$. It is considered that epigenetic factors play a critical role in tooth development that can create variability at all phenotypicalsomatic and behavioral levels. For example, histone demethylase regulate the differentiation of dental stem cell ${ }^{1}$. Furthermore, it is supposed that epigenetic influences the spatial arrangement of cells and the timing of the interactive signaling, contributing to differences in tooth size and shape, number and dental asymmetry in MZ co-twins ${ }^{1,27}$. Maybe there exited certain epigenetic influences which lead to the differences of phenotype between the probed and her brother. Thus, a further study of epigenetic biomarkers is needed to explain the reasons for observed differences.

Moreover, there may be other as-yet-unknown factors associated with severe anodontia and relevant to severe non-syndromic anodontia in the Chinese population. Therefore, it is still a question why the same genotype can lead to different phenotypes. In view of this multifactorial nature of dental anomalies, specific mutations of single genes and their acting background should be explored. The genotype/phenotype correlation in congenital anodontia cannot be verified, as there was only one pedigree analyzed. So, further genotype/phenotype investigations are required in the future.

\section{ACKNOWLEDGMENTS}

We thank the research subjects for their participation in the research. We are also grateful to Dr. Kun Zhang and Ying Shen for their technical assistance and collaboration. This study was financially supported by the Science \& Technology Department of Sichuan Province (2010FZ0042), Jiangsu Natural Science Foundation (BK2007008) and NSFC (National Natural Science Foundation of China) No. 81070858 and 81100778.

\section{REFERENCES}

1- Brook AH. Multilevel complex interactions between genetic, epigenetic and environmental factors in the aetiology of anomalies of dental development. Arch Oral Biol. 2009;54:S3-17.

2- Chranowska KH, Krajewska-Walasek M, Rump Z, Wisniewski L, Fryns JP. Anodontia as the sole clinical sign of the ectrodactylyectodermal dysplasia-cleft lip (EEC) syndrome. Genet Counse. $1990 ; 1: 67-73$.

3- De Coster PJ, Marks LA, Martens LC, Huysseune A. Dental agenesis: genetic and clinical perspectives. J Oral Pathol Med. 2009;38:1-17.

4- De Coster PJ, Mortier G, Marks LA, Martens LC. Cranial suture biology and dental development: genetic and clinical perspectives. J Oral Pathol Med. 2007;36:447-55.

5- Fryns JP, Legius E, Dereymaeker AM, Van den Berghe H. EEC syndrome without ectrodactyly: report of two new families. J Med Genet. 1990;27:165-8.

6- Gerits A, Nieminen P, De Muynck S, Carels C. Exclusion of coding region mutations in MSX1, PAX9 and AXIN2 in eight patients with severe oligodontia phenotype. Orthod Craniofac Res. 2006;9:129-36.

7- Goldenberg M, Das P, Messersmith M, Stockton DW, Patel PI, D'Souza RN. Clinical, radiographic, and genetic evaluation of a novel form of autosomal-dominant oligodontia. J Dent Res. 2000;79:1469-75. 
8- Han D, Gong Y, Wu H, Zhang X, Yan M, Wang X, et al. Novel EDA mutation resulting in $X$-linked non-syndromic hypodontia and the pattern of EDA-associated isolated tooth agenesis. Eur J Med Genet. 2008;51:536-46.

9- Hoo JJ. Anodontia of permanent teeth (OMIM \# 206780) and pegged/missing maxillary lateral incisors (OMIM \# 150400) in the same family. Am J Med Genet. 2000;90:326-7.

10- Jiang $X$, Wu G, Zhou J, Wang S, Tseng AA, Du Z. Nanopatterning on silicon surface using atomic force microscopy with diamond-like carbon (DLC)-coated Si probe. Nanoscale Res Lett. 2011;6:518. 11- Kangas AT, Evans AR, Thesleff I, Jernvall J. Nonindependence of mammalian dental characters. Nature. 2004;432:211-4.

12- Line SR. Variation of tooth number in mammalian dentition: connecting genetics, development, and evolution. Evol Dev. 2003; 5:295-304.

13- Matalova E, Fleischmannova J, Sharpe PT, Tucker AS. Tooth agenesis: from molecular genetics to molecular dentistry. J Dent Res. 2008;87:617-23.

14- Mostowska A, Biedziak B, Jagodzinski PP. Axis inhibition protein 2 (AXIN2) polymorphisms may be a risk factor for selective tooth agenesis. J Hum Genet. 2006;51:262-6.

15- Mostowska A, Kobielak A, Trzeciak WH. Molecular basis of non-syndromic tooth agenesis: mutations of MSX1 and PAX9 reflect their role in patterning human dentition. Eur J Oral Sci. 2003; 111:365-70.

16- Nakatomi M, Wang XP, Key D, Lund JJ, Turbe-Doan A, Kist $\mathrm{R}$, et al. Genetic interactions between $P A X 9$ and $M S X 1$ regulate lip development and several stages of tooth morphogenesis. Dev Biol. 2010;340:438-49.

17- Ogawa T, Kapadia H, Feng JQ, Raghow R, Peters H, D'Souza RN. Functional consequences of interactions between $P A X 9$ and MSX1 genes in normal and abnormal tooth development. J Biol Chem. 2006;281:18363-9.

18- Ogawa T, Kapadia H, Wang BL, D'Souza RN. Studies on Pax9Msx1 protein interactions. Arch Oral Biol. 2005;50:141-5.

19- Online Mendelian Inheritance in Man [homepage]. Baltimore: Johns Hopkins University; c1966-2012 [cited 25 Apr. 2013]. Available from: http://www.omim.org/.

20- Paixao-Côrtes VR, Braga T, Salzano FM, Mundstock K, Mundstock CA, Bortolini MC. PAX9 and MSX1 transcription factor genes in non-syndromic dental agenesis. Arch Oral Biol. 2011;56:337-44

21- Paixão-Côrtes VR, Meyer D, Pereira TV, Mazières S, Elion J, Krishnamoorthy $\mathrm{R}$, et al. Genetic variation among major human geographic groups supports a peculiar evolutionary trend in $P A X 9$. PLoS One. 2011;6:e15656.
22- Pan $Y$, Wang L, Ma J, Zhang W, Wang M, Zhong W, et al. PAX9 polymorphisms and susceptibility to sporadic tooth agenesis: a case-control study in southeast China. Eur J Oral Sci. 2008; 116:98-103.

23- Pawlowska E, Janik-Papis K, Poplawski T, Blasiak J, Szczepanska J. Mutations in the PAX9 gene in sporadic oligodontia. Orthod Craniofac Res. 2010;13:142-52.

24- Peres RC, Scarel-Caminaga RM, Espirito Santo AR, Line $\mathrm{SR}$. Association between $P A X-9$ promoter polymorphisms and hypodontia in humans. Arch Oral Biol. 2005;50:861-70.

25- Song S, Han D, Qu H, Gong Y, Wu H, Zhang X, et al. EDA gene mutations underlie non-syndromic oligodontia. J Dent Res. 2009;88:126-31.

26- Tao R, Jin B, Guo SZ, Qing W, Feng GY, Brooks DG, et al. A novel missense mutation of the EDA gene in a Mongolian family with congenital hypodontia. J Hum Genet. 2006;51:498-502.

27- Townsend G, Hughes T, Luciano M, Bockmann M, Brook A. Genetic and environmental influences on human dental variation: a critical evaluation of studies involving twins. Arch Oral Biol. 2009;54(Suppl 1):S45-51.

28- Vastardis $\mathrm{H}$. The genetics of human tooth agenesis: new discoveries for understanding dental anomalies. Am J Orthod Dentofacial Orthop. 2000;117:650-6

29- Vieira AR, Meira R, Modesto A, Murray JC. MSX1, PAX9, and TGFA contribute to tooth agenesis in humans. J Dent Res. 2004;83:723-7.

30- Wang J, Jian F, Chen J, Wang H, Lin Y, Yang Z, et al. Sequence analysis of $P A X 9, M S X 1$ and $A X I N 2$ genes in a Chinese oligodontia family. Arch Oral Biol. 2011;56:1027-34.

31- Witkop CJ Jr. Agenesis of succedaneous teeth: an expression of the homozygous state of the gene for the pegged or missing maxillary lateral incisor trait. Am J Med Genet. 1987;26:431-6. 32- Xuan K, Jin F, Liu YL, Yuan LT, Wen LY, Yang FS, et al. Identification of a novel missense mutation of $M S X 1$ gene in Chinese family with autosomal-dominant oligodontia. Arch Oral Biol. 2008;53:773-9.

33- Zhang YD, Chen Z, Song YQ, Liu C, Chen YP. Making a tooth: growth factors, transcription factors, and stem cells. Cell Res. 2005;15:301-16.

34- Zhao JL, Hu QG, Chen YX, Luo SJ, Bao L, Xu Y. A novel missense mutation in the paired domain of human $P A X 9$ causes oligodontia. Am J Med Genet A. 2007;143A:2592-7. 